\title{
Indexing Tree Structures through Caterpillar Decomposition
}

\author{
Fadi Yilmaz and M. Fatih Demirci \\ TOBB University of Economics and Technology, \\ Computer Engineering Department, \\ Sogutozu Cad. No:43, 06560 Ankara, Turkey \\ \{fhyilmaz,mfdemirci\}@etu.edu.tr
}

\begin{abstract}
Graphs provide effective data structures modeling complex relations and schemaless data such as images, XML documents, circuits, compounds, and proteins. Given a query graph, efficiently finding all database graphs in which the query is a subgraph is an important problem raising in different domains. In this paper, we propose a new method for indexing tree structures based on a graph-theoretic concept called caterpillar decomposition and discuss its advantages over two previous indexing algorithms. Experimental evaluation of the proposed framework including the comparison with the previous approaches demonstrates the efficacy of the overall approach.
\end{abstract}

Keywords: shape retrieval, indexing, caterpillar decomposition.

\section{Introduction}

One of the highly active research areas within the field of multimedia systems is multimedia retrieval. With the increasing availability of multimedia collections due to various digital storage devices, an efficient retrieval of similar multimedia items for a given query from a large database is essential. Since images form the base for other multimedia types such as video and animation, we present an efficient graph-based image retrieval system in this paper.

Graphs provide an effective data structure modeling complex relations such as organization of entities in images, XML documents, compounds, and proteins. A critical and common retrieval problem exists in many graph-based applications. Given a query graph $q$ and database $D=\left\{g_{1}, g_{2}, \ldots, g_{n}\right\}$, this problem is stated as efficiently finding all graphs in which $q$ is a subgraph. Since checking whether a pair of graphs is isomorphic to each other is computationally expensive, sequential search of the database for this problem is impractical.

A number of indexing approaches have been proposed in the past to address this problem. Generally, these approaches use some graph attributes such as maximum, minimum, average node degrees, path lengths, and node adjacency to locate database graphs with similar attributes. One such approach presented in the literature is path-based approach 8 87/2, which utilizes graph paths for indexing. In particular, this algorithm first extracts paths from each database

A. Heyden and F. Kahl (Eds.): SCIA 2011, LNCS 6688, pp. 687-696, 2011.

(C) Springer-Verlag Berlin Heidelberg 2011 


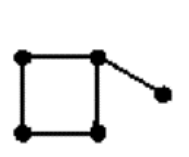

(a)

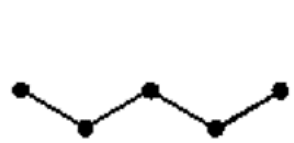

(b)

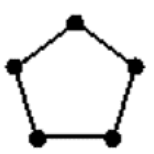

(c)

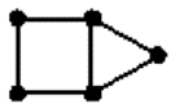

(d)

Fig. 1. Limitations of the path-based approach. Although the query shown in part (a) appears as a subgraph in part (d) only, the path-based approach retrieves all graphs in parts (b)-(d)

graph up to same maximum length. Given a query graph, it then locates a database graph, which contains all paths that exist in the query. Although one can easily index a query graph into the database using the path-based approach, this indexing algorithm has an important drawback: paths of a graph do not carry sufficient information regarding its structure, resulting in a number of false positive retrievals. Figure 1 presents an example, where query and three database graphs are shown in parts (a)-(d), respectively. One may notice that the only graph in part (d) includes the query as a subgraph. However, since all database graphs contain a path up to length four, they are all retrieved as the result of this indexing algorithm. This shows that the path-based approach is not suitable for applications where graphs in the system contain too many paths.

To decrease the number of false positive retrievals, an indexing algorithm should take into consideration graph's structure. gIndex 11] is an example of this type of indexing. This algorithm extracts frequent subgraphs from the database and uses discriminative ones for indexing. Here, a subgraph is called frequent if it is included by a large number of database graphs. Each frequent and discriminative substructures form the feature set. Once the feature set is computed, features are translated into sequences and a prefix tree is constructed to store and retrieve them efficiently. For a given query, all its substructures up to some maximum size is generated and the prefix tree is used to search for database graphs which consists of the same substructures.

In this paper, we propose a new method for indexing tree structures. Our algorithm is based on a graph-theoretic concept named caterpillar decomposition (CD), which is the collection of edge-disjoint, root-leaf paths. Computing the $\mathrm{CD}$ for a tree enables us to represent it as a vector in the geometric space. After representing the query in the same fashion, we perform a range search around the query to retrieve similar database trees efficiently. The proposed algorithm uses tree structures for indexing rather then path lengths alone and it does not require an extra step to determine frequent substructures unlike path-based and gIndex approaches. Experimental evaluation of the proposed approach shows the improved retrieval performance over these two algorithms. Figure 2 presents an overview of the proposed framework.

Indexing tree structures has been proposed before by Shokoufandeh et al. [10]. In that work, the sum of the largest eigenvalues of the tree's adjacency matrix for the root is used to represent it in the geometric space. After performing the same process for the query, similar database trees is retrieved by means 

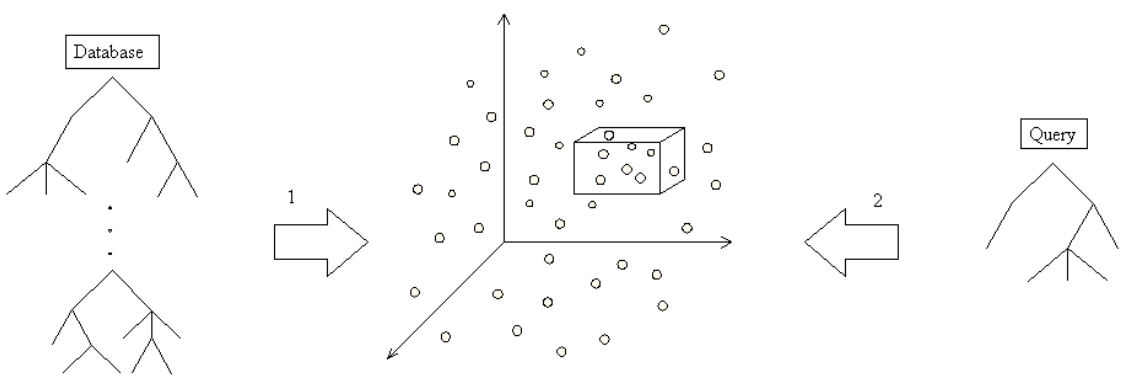

Fig. 2. Overview of the proposed algorithm. Each database tree is represented as a set of points in the geometric space using its caterpillar decomposition (transition 1). After representing query in the same fashion (transition 2), trees with the same substructures are retrieved by means of an efficient k-nearest neighbor search algorithm.

of an efficient nearest neighbor search around the query vector. To account for local deformation and substructure, this approach also represents the root of each subtree as such a vector. Eigenvalues of graph's laplacian matrices has also been used for indexing in the literature [3]. The motivation for choosing laplacian matrix over adjacency matrix comes from studies showing that the laplacian matrix is more informative and more representative in terms of creating fewer number of co-spectral graphs. Given a graph, this approach computes its signature using the sorted eigenvalues of laplacian matrix. For a query graph and a large database, the indexing amounts to a nearest neighbor search in the model database. Similar to the work presented in [10], the authors compute the vectors for each subgraph in the system. Borrowing the same ideas, we compute the CD for each subtree to represent the local structure and thus to retrieve graphs with the same substructures.

The rest of the paper is organized as follows. After providing a brief review of some concepts in Section 2, we describe our indexing approach in Section 3. We present the experiments including the comparison of our approach with pathbased and gIndex algorithms in the domain of shape retrieval in Section 4 . Finally, we finish the paper with the conclusion in Section 5 .

\section{Preliminaries}

In this section, we provide definitions of some concepts which we use in our framework. A graph $G$ is a pair $(V, E)$, where $V$ is a finite set of vertices (nodes) and $E$ is a set of edges between the vertices. An edge $e=(u, v)$ connects two vertices such that $u, v \in V$. Two vertices are adjacent, if there exists an edge between them. $|G|$ denotes the size of graph $G$ and it is defined by the number of vertices. A tree is a graph without cycles. A pair of vertices in the tree is, thus, connected by one simple path. 


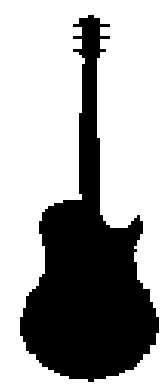

(a)

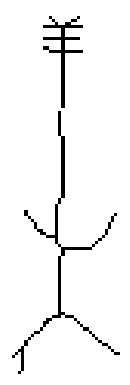

(b)

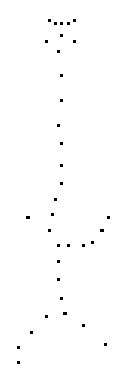

(c)

Fig. 3. Skeleton points of the silhouette image in part (a) is shown in part (b). After applying k-means algorithm, the representative points are shown in part (c). The minimum spanning tree is computed using the representative points.

Two graphs $G_{1}$ and $G_{2}$ share substructures if there exists two subgraphs $g_{1} \in$ $G_{1}$ and $g_{2} \in G_{2}$ which are isomorphic to each other. Two graphs $H=\left(V_{H}, E_{H}\right)$ and $G=\left(V_{G}, E_{G}\right)$ are said to be isomorphic if there is a bijection between their vertex sets, $f: V_{H} \rightarrow V_{G}$ such that any pair of vertices $u$ and v of $G$ are adjacent if and only if $f(u)$ and $f(v)$ are adjacent in $H$.

Since the proposed indexing framework is designed especially for trees, our algorithm starts by representing input images as trees through skeleton points. A skeleton point (or, shock point) is defined in 4 as the dynamic view of the medial axis where the propagation of waves from the shape boundary results in the formation of singularities. In [1] medial axis is described as the locus of centers of circles inside the region which are bitangent to the boundary in at least two places. Each skeleton point $p$ is associated with a 3 -dimensional vector $v(p)=(x, y, r)$, where $(x, y)$ are the Euclidean coordinates of the point and $r$ is the radius of the maximal bi-tangent circle centered at the point. Each shock point represented as a vertex in the skeleton graph, which takes over significant role especially on structural and statistical pattern recognition. Each pair of skeleton points in the graph is connected by an edge whose weight reflects the Euclidean distance between them. We convert the graph to a tree by computing its minimum spanning tree. As a result, nodes correspond to skeleton points, and edges connect nearby skeleton points. The root of the tree is the node that minimizes the sum of the shortest path distances to all other nodes.

The number of the skeleton points is proportional to size of the image. As a result, the large number of points make the overall process slower. To reduce the number of these points, we use k-means algorithm, which clusters the skeleton points and returns one representative for each cluster. The minimum spanning tree is then computed using the k-representatives. Figure 3 illustrates this process. The skeleton points of a silhouette image shown in part (a) appear in part (b). Part (c) shows the k-means cluster representatives computed using these points. 


\section{Indexing Tree Structures through Caterpillar Decomposition}

Given a query and a large database represented as tree structures, the objective of our indexing algorithm is efficient retrieval of database trees, which share substructures with the query. Our algorithm is based on a graph-theoretical concept named caterpillar decomposition (CD), which captures the topological structure of the tree. This concept has been used in [56] for embedding the tree nodes into low dimensional Euclidean spaces such that the distances between the vertices are realized by the Euclidean distances between the embedded points. In this paper, we use the CD to represent the root of each input tree as a vector. Once each tree is represented as such a vector, performing a nearest neighbor search around the query allows us to retrieve trees consisting of the same substructures.

The concept of the CD is described in a sample tree shown in Figure 4. The three paths between $a$ and $e, a$ and $j, a$ and $h$ are called level 1 paths and represent first three paths in caterpillar decomposition. If we remove these three level 1 paths from the tree, we are left with the 2 edge-disjoint paths. These are the paths between $c$ and $k$, and $b$ and $l$, called level 2 paths, which represent the other two paths in caterpillar decomposition. If removing the level 2 paths had left additional connected components, the process would be repeated until all the edges in the tree had been removed. The union of the paths is called the caterpillar decomposition of the tree. The total number of paths in the CD specifies the dimensionality of the geometric space into which the root of the tree is embedded.

To compute the coordinate of the root in the geometric space, we begin by finding the unique path from each leaf lying on the first level. The weight of a root-leaf path determines the value of its corresponding coordinate. Once the weight of each path on the first level is computed, we repeat this process with the leaves on the second level, etc., until all leaves of the tree have been considered. In this procedure, the order in which the paths appear in CT changes the position of the root in the geometric space. To be consistent, we select the paths by their

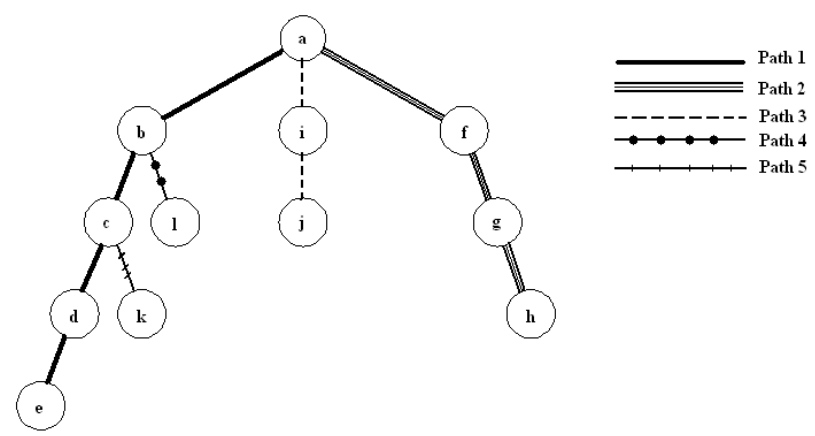

Fig. 4. Caterpillar decomposition of a rooted tree. Root-leaf paths are extracted from the tree by their levels and weights. 
levels and their weights. To illustrate this algorithm, we turn back to Figure 4 in which the root of the tree is embedded into a 5-dimensional space. Assuming that each edge in the tree has a uniform weight, the value of the first coordinate is determined by the weight between $a$ and $e$, which is 4 in this case. The value of the second and the third coordinates are also defined by the paths from the leaves on the first level, namely $h$ and $j$, respectively. Repeating the same process for the level 2 paths, we obtain the coordinates of the root, $(4,3,2,3,2)$.

It is important to note that using the above procedure, trees are represented into geometric spaces of different dimensions. Therefore, we first have to perform a registration step whose objective is to represent the trees in the same space. To do this, we bring up lower dimensional signatures to higher dimensions by padding them with zeros. Let $p_{m}$ denote the maximum number of paths in a database tree. Suppose that tree $T_{1}$ has $p_{1}$ paths and $p_{1} \leq p_{m}$. By adding $p_{m}-p_{1}$ 0 -valued coordinates, we make the dimensions of the tree signatures equal. In case that the number of paths in the CD of the query is greater than $p_{m}$, we reduce its dimension using a dimensionality reduction technique, e.g., Principal Component Analysis.

Having equalized the dimensionality of the trees and represented them in the same space, we can now proceed with performing a nearest neighbor search around the query. Unfortunately, the indexing formulation given above cannot support local structures: two trees may share the same structures up to only some level. Although adding or removing tree structure changes the coordinates of the root, the position for the root of each subtree that survives such alteration will not be affected. Therefore, the proposed indexing mechanism cannot depend on the vector (or, signature) of the whole tree only. As done in previous indexing algorithms [103], we compute the signature of each subtree in the database and represent each signature in the geometric space using the same fashion.

Applying the same process to the given query results in a set of vectors corresponding to the tree and subtrees of the query. We then perform a nearest neighbor search around each vector and combine their retrieval results in a weighted schema such that database trees with bigger size and closer distance to the query vectors gets higher weights then others. More specifically, let $S_{Q}=\left\{s_{q 1}, \ldots, s_{q m}\right\}$ be the set of query signatures extracted from query $Q$. For a particular signature $s_{q i} \in S_{Q}$, let $N_{s_{q i}}=\left\{s_{t 1}, \ldots, s_{t k}\right\}$ be the set of database signatures returned by the nearest neighbor search. We compute the weight of the vote between $s_{q i}$ and a signature $s_{t i}$ computed from database (sub)tree $t_{i}$ as follows:

$$
\psi_{s_{q i}, s_{t i}}=\frac{\left|t_{i}\right|}{1+\left\|s_{q i}-s_{t i}\right\|_{2}}
$$

Let $S_{T}=\left\{s_{t 1}, \ldots, s_{t p}\right\}$ denote the set of all signatures for one particular database tree $T$. The similarity between between query signature $s_{q i}$ and $s_{t l} \in S_{T}$ is computed as follows:

$$
w_{s_{q i}, s_{t l}}= \begin{cases}\psi_{s_{q i}, s_{t l}} & \text { if } s_{t l} \in N_{s_{q i}} \\ 0 & \text { otherwise. }\end{cases}
$$




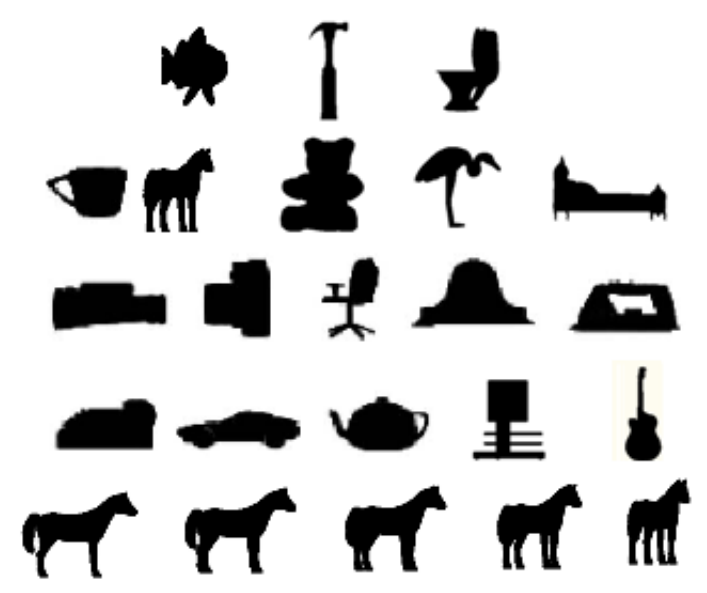

Fig. 5. The top four row presents sample silhouettes from the dataset, while the bottom row shows some sample views for the same object class

Given a query $Q$ and database tree $T$ along with their signature sets $S_{Q}=$ $\left\{s_{q 1}, \ldots, s_{q m}\right\}$ and $S_{T}=\left\{s_{t 1}, \ldots, s_{t p}\right\}$ computed from $Q$ and $T$, respectively, the final similarity between $Q$ and $T$ is then obtained as:

$$
W_{Q, T}=\sum_{u=1}^{m} \sum_{v=1}^{p} w_{s_{q u} s_{t v}} .
$$

This formulation ensures that database trees which frequently appear in the nearest neighbor lists of the query get higher similarity scores than others.

Revisiting the key features of the proposed algorithm, encoding of a tree's structure captures its local topology, thus allowing for its use to retrieve database trees with the same substructures. The signature of a tree is invariant under the reorderings of its branches. This, in turn, allows us to compare the signatures of a pair of trees without solving the corresponding problem. In addition, the algorithm sorts the database trees by similarity to the given query without requiring an extra step to determine the frequent substructures.

\section{Experiments}

In this section we evaluate the proposed approach in the context of a shape recognition experiment. We use a silhouette dataset, consisting of 18 different objects with 72 views for each. The top four rows of Figure 5 presents sample silhouettes, while the bottom row shows some sample views for the same object. Each silhouette in the dataset is represented as a rooted undirected skeleton tree using the method described in Section 2 .

To test the proposed approach on the database, we removed 36 of the 72 views of each object (every other view) and used these as queries to the remaining 
Table 1. Retrieval results for path-based, gIndex, and the proposed algorithms. NN: nearest neighbor, FT: first tier, ST: second tier. The proposed algorithm outperforms the two previous algorithms for these three criteria.

\begin{tabular}{|c||c|c|c|}
\hline & $\mathrm{NN}(\%)$ & $\mathrm{FT}(\%)$ & $\mathrm{ST}(\%)$ \\
\hline PATH-BASED & 38.9 & 32.6 & 61.6 \\
\hline GINDEX & 57.8 & 50.5 & 71.4 \\
\hline PROPOSED WORK & 91.2 & 66.7 & 88.3 \\
\hline
\end{tabular}

database, which is the other 36 views for each of the 18 objects. We then performed nearest neighbor searches around each query vector to retrieve database trees with the same substructures. We computed nearest neighbor (NN), first tier $(\mathrm{FT})$, and second tier (ST) retrieval rates for the experiments. Here, the first tier rate measures the number of the models in the query's class appearing within the top $K-1$ retrievals, where $K$ denotes the size of the query's class 9 . Similarly, the second tier considers the top $2 K-1$ retrievals for measuring the same rate. For comparison purposes, we also computed the retrieval rates of both path-based and gIndex approaches on the same database.

The results are shown in Table 1 and reveal that the proposed framework and gIndex algorithm are more effective than the path-based approach for all three retrieval rates. This shows the importance of encoding structural information for retrieval rather than using the path lengths only. One may notice that the proposed framework also outperforms the gIndex algorithm considering all three retrieval criteria. Although both approaches take into consideration tree structures, the gIndex algorithm look for exact patterns that exist in the query. In case a minor topological change to a tree happens, e.g., slightly changing the edge weights or adding/removing a leaf, exact patterns may not appear in close structures. On the other hand, these small changes make the coordinate for the root of the resulting tree close to its original position using our algorithm. As a result, representing substructures by computing the CD for each subtree in the system enables the proposed approach to retrieve trees with similar substructures effectively.

To test the sensitivity of the proposed indexing algorithm to perturbation of the query, we perturbed each query by deleting a randomly selected connected subset of its skeleton points whose size was around $15 \%$ of the total number of skeleton points. The average nearest neighbor, first tier, and second tier retrieval rates were recorded as $85.7 \%, 60.8 \%, 83.3 \%$, respectively. This reflects the algorithm's stability to missing data. Although not a true occlusion experiment, these results present the algorithm's ability to match local structure.

We should note that many of the objects in the database are symmetric. If a query has an identical view elsewhere on the object, that view might be retrieved as its nearest neighbor and scored as an error. Thus, the nearest neighbor rates, in these experiments, should be considered as worst-case. In addition, by improving the sampling resolutions of the viewing sphere, we expect that retrieval rates would increase for all three algorithms. 


\section{Conclusions}

Graph indexing is an important problem facing researchers in different domains. In this paper, we have proposed a new technique for indexing tree structures using a graph-theoretical concept, named caterpillar decomposition, which is defined as the collection of edge-disjoint, root-leaf paths. Our algorithm starts by representing each database tree along with its subtrees as a point in the geometric space based on its caterpillar decomposition. After representing the query in the same fashion, an efficient retrieval of trees with the same substructures is performed by means of a nearest neighbor search. Experimental evaluation of the framework, including a comparison with the two previous approaches demonstrates the efficacy of the overall algorithm. Performing a more comprehensive experimental test using a larger dataset with different image formats and applying our framework to different domains are our future plans.

\section{Acknowledgements}

Fatih Demirci gratefully acknowledges the support of TÜBİTAK Career grant 109E183.

\section{References}

1. Blum, H.: Biological shape and visual science (part i). Journal of Theoretical Biology 38(2), 205-287 (1973)

2. Chen, Q., Lim, A., Ong, K.: D(k)-index: an adaptive structural summary for graphstructured data. In: Proceedings of the 2003 ACM SIGMOD International Conference on Management of Data, pp. 134-144. ACM, New York (2003)

3. Demirci, M.F., van Leuken, R.H., Veltkamp, R.C.: Indexing through laplacian spectra. Computer Vision Image Understanding 110(3), 312-325 (2008)

4. Giblin, P., Kimia, B.: On the local form and transitions of symmetry sets, medial axes, and shocks. International Journal of Computer Vision 54(1-3), 143-156 (2003)

5. Gupta, A.: Embedding tree metrics into low dimensional euclidean spaces. In: Proceedings of the Thirty-First Annual ACM Symposium on Theory of Computing, pp. 694-700. ACM, New York (1999)

6. Matousek, J.: On embedding trees into uniformly convex banach spaces. Israel Journal of Mathematics 237, 221-237 (1999)

7. Min, J., Chung, C., Shim, K.: An adaptive path index for xml data using the query workload. Information Systems 30(6), 467-487 (2005)

8. Shasha, D., Wang, J., Giugno, R.: Algorithmics and applications of tree and graph searching. In: Proceedings of the 21st ACM SIGMOD-SIGACT-SIGART Symposium on Principles of Database Systems, Madison, Wisconsin, pp. 39-52. ACM, New York (2002)

9. Shilane, P., Min, P., Kazhdan, M., Funkhouser, T.: The princeton shape benchmark. In: Proceedings of the Shape Modeling International, pp. 167-178. IEEE Computer Society, Washington, DC, USA (2004) 
10. Shokoufandeh, A., Macrini, D., Dickinson, S., Siddiqi, K., Zucker, S.W.: Indexing hierarchical structures using graph spectra. IEEE Transactions on Pattern Analysis and Machine Intelligence 27(7), 1125-1140 (2005)

11. Yan, X., Yu, P., Han, J.: Graph indexing: a frequent structure-based approach. In: Proceedings of the 2004 ACM SIGMOD International Conference on Management of Data, Paris, France, pp. 335-346. ACM, New York (2004) 